\title{
LINEAR REPRESENTATIONS OF TOPOLOGICAL GROUPS
}

\section{MORIKUNI GOTÔ}

Introduction. In his recent papers entitled Faithful representations of Lie groups, I and II, ${ }^{1}$ the author has called a locally compact connected group $L$ faithfully representable (f.r.) if $L$ admits a faithful representation, ${ }^{2}$ and has determined the characteristic properties of f.r. groups. It is to be pointed out that although an f.r. group is a Lie group, a connected Lie group is not always f.r. ${ }^{3}$ Furthermore, he introduced there a notion of $(l)$-groups, and proved that a Lie group is f.r. if it is an (l)-group, ${ }^{4}$ where the definition of $(l)$-groups runs as follows:

DeFINITION 1. Let $G$ be a locally compact connected group. Then $G$ is called an ( $l$ )-group if for every element $g$ of $G$ distinct from the identity there exists a representation of $G$ under which $g$ does not go over into the unit matrix.

The above definition is obviously equivalent to the one of imposing on $G$ the condition that $G$ can be approximated by f.r. groups, namely that there exists a system $\left\{N_{\alpha}\right\}$ of closed invariant subgroups of $G$ so that every factor group $G / N_{\alpha}$ is f.r. and the intersection of all $N_{\alpha}$ 's coincides with the identity group $\{e\}$, which is composed of the identity $e$ only.

In the present paper we shall study the structure of $(l)$-groups as a sequel of [M.G.]. Now K. Iwasawa called a locally compact group $G$ an (L)-group if $G$ can be approximated by Lie groups; he studied the structure of $(L)$-groups, and in particular solved the socalled fifth problem of Hilbert for such groups. ${ }^{5}$ Our $(l)$-group is of course a connected $(L)$-group and our study is based naturally on the theory of $(L)$-groups also.

Next we shall state the outline of the present paper. In $\$ 1$ we study semi-simple $(L)$-groups, where the semi-simpleness of an $(L)$-group is defined in an analogous way as in the theory of Lie groups. \$2 is

Received by the editors May 3, 1949.

1 Mathematica Japonicae vol. 1 (1949), and Nagoya Mathematical Journal vol. 1 (1950). Referred to as [M.G.], or explicitly [M.G.I.] or [M.G.II.].

2 By a representation we mean a continuous linear representation. An f.r. group can also be defined as a topological group topologically isomorphic with a closed connected subgroup of the general linear group. [M.G.II, Theorem 9].

See, for example [M.G.].

- See [M.G.II, Theorem 8].

- K. Iwasawa, On some types of topological groups, Ann. of Math. vol. 50 (1949). We shall refer to this paper as [K.I.]. 
devoted to the proof of Theorem 2, which has an essential bearing on the latter part of this paper. Now in $\$ 3$ we deal with the class of completely reducible (l)-groups, which contains both compact connected groups and semi-simple (l)-groups. After such preparations we shall investigate in $\$ 4$ the structure of general $(l)$-groups. Theorem 5 , as well as Theorem 6 , is our fundamental result, affording a characterization of $(l)$-groups. Lastly in $\$ 5$ some supplementary facts about solvable $(l)$-groups are stated.

In closing the introduction, the author wishes to express his sincere gratitude to Mr. Y. Matsushima for his valuable suggestions and cooperation.

1. Semi-simple ( $L$ )-groups. Let $G$ be a locally compact group. $G$ contains then the radical, that is, the uniquely determined maximal connected solvable invariant subgroup, which is closed in $G$ in virtue of [K.I.]. Now we shall extend the notion of semi-simpleness of Lie groups to $(L)$-groups, as follows:

DEFINITION 2. An $(L)$-group is called semi-simple if its radical coincides with the identity group.

Since the radical is characteristic, any closed invariant subgroup of a semi-simple $(L)$-group is also semi-simple.

Now in order to study the structure of semi-simple $(L)$-groups we first give the following two lemmas.

LeMma 1. Let $G$ be a locally compact connected group, and let $C$ and $N$ be closed invariant subgroups of $G$ such that $G=C N$. Denote by $C^{0}$ and $N^{0}$ the connected components of $C$ and $N$ containing the identity respectively. Suppose $C$, or $C^{0}$, be compact, then we have $G=C^{0} N^{0}$.

Proof. As $C^{0}$ is compact, $C^{0} N$ is a closed invariant subgroup. Since the intersection of $C^{0} N$ and $C$ contains $C^{0}$, the isomorphism ${ }^{6} G / C^{0} N$ $\cong C / C^{0} N \cap C$ implies that $G / C^{0} N$ is 0 -dimensional. On the other hand, $G / C^{0} N$ must be connected because of the connectedness of $G$. Hence we get $G=C^{0} N$. Now a similar argument as above would establish the lemma. Q.E.D.

LeMma 2. Let $G$ be a connected semi-simple $(L)$-group, and $K$ a compact 0-dimensional invariant subgroup of $G$. Suppose that the factor group $G / K$ is a Lie group. Then $G$ itself is a Lie group.

Proof. It is clearly sufficient to prove that $K$ cannot be infinite and separable. Suppose that $K$ be an infinite separable group. Then

\footnotetext{
- By an isomorphism (or a homomorphism) we mean a continuous open isomorphism (or homomorphism).
} 
there exists a sequence $\left\{K_{n}\right\}$ :

$$
K=K_{1} \supset K_{2} \supset K_{3} \supset \cdots, \quad \Psi_{n} \neq K_{n+1},
$$

of open subgroups of $K$ such that $\cap K_{n}=\{e\}$. Since $K$ is a central invariant subgroup and any open subgroup of a topological group is closed, $K_{n}$ 's are all closed invariant subgroups.

We denote by $\bar{G}$ the universal covering group of $G / K$. Then we can readily select a sequence $\left\{Z_{n}\right\}$ of central invariant subgroups of $\tilde{G}$ :

$$
Z_{1} \supset Z_{2} \supset Z_{3} \supset \cdots, \quad Z_{n} \neq Z_{n+1},
$$

such that $\tilde{G} / Z_{n} \cong G / K_{n}, n=1,2, \cdots$. Denote by $Z$ the intersection of all $Z_{n}$. Then it is easy to construct an algebraic isomorphism between $G / Z$ and $G$. Since $K$ is central, $K$ is a countable group. On the other hand, it is easy to prove that a countable compact group is discrete. Hence $K$ must be finite, contrary to the hypothesis. This completes our proof.

Now let $G$ be a connected semi-simple $(L)$-group and let $C^{*}$ be the (uniquely determined) maximal compact invariant subgroup ${ }^{7}$ of $G$. Then by a theorem of Iwasawa in [K.I.], $G$ contains a closed invariant subgroup $L^{*}$ of $G$ such that $G=C^{*} L^{*}$ and $\left[C^{*}, L^{*}\right]=\{e\}$, where we denote by $\left[C^{*}, L^{*}\right]$ the subgroup generated by the elements of the form $c^{-1} l^{-1} c l$ with $c \in C^{*}$ and $l \in L^{*}$. Let $C$ and $L$ be the connected components of $C^{*}$ and $L^{*}$ respectively containing the identity. Then, using Lemma 1 , we obtain that $G=C L$.

Let now $K$ be the maximal compact invariant subgroup of $L$, and $K^{0}$ the connected component of $K$ containing $e$. $C$ being the maximal connected compact invariant subgroup, $K^{0}$ must be contained in the intersection of $C$ and $L$. Hence $K^{0}$ is central. This shows that $K^{0}$ coincides with the identity group, whence $K$ is 0 -dimensional. Therefore, using Lemma 2, we get the fact that $L$ is a Lie group. Now denote by $D$ the intersection of $C$ and $L$. Since $D$ is compact and is contained in the center of $L, D$ must be a finite group.

Next let $\left\{\widetilde{C}_{\lambda}\right\}$ be a sequence of compact connected simply connected semi-simple simple Lie groups, and $\tilde{C}$ the direct product of all $\tilde{C}_{\lambda}$ 's $: \widetilde{C}=\prod \tilde{C}_{\lambda} . \tilde{C}$ is also a compact group with respect to the so-called weak topology. Now let $D$ be a closed 0-dimensional invariant subgroup of $\tilde{C}$. Then the factor group $\tilde{C} / D$ is obviously semi-simple, and conversely any connected compact semi-simple group is readily

${ }^{7}$ The existence, as well as the uniqueness, of such a subgroup for any connected (L)-group has been assured in [K.I.]. 
proved to be obtained in such a way. ${ }^{8}$

In a similar way, we can establish a method of constructing all connected semi-simple $(L)$-groups: Let $\left\{\widetilde{S}_{\lambda}\right\}$ be a sequence of connected simply connected semi-simple simple Lie groups such that almost all of $\tilde{S}_{\lambda}$ 's are compact, and let $\tilde{S}$ be the direct product of all $\tilde{S}_{\lambda}$ 's: $\widetilde{S}=\prod \widetilde{S}_{\lambda}$. Then $\widetilde{S}$ is clearly a connected semi-simple $(L)$-group with respect to the weak topology. Now let $D$ be a closed 0 -dimensional invariant subgroup of $\widetilde{S} . \widetilde{S} / D$ is clearly semi-simple, and any connected semi-simple $(L)$-group is isomorphic with some $\prod \tilde{S}_{\lambda} / D$ for suitable $\left\{\widetilde{S}_{\lambda}\right\}$ and $D$.

Now a factor group of the above $\Pi \widetilde{S}_{\lambda}$ is clearly semi-simple. Hence any factor group of a connected semi-simple $(L)$-group is also semi-simple. Thus we get the following theorem.

Theorem 1. (1) Let $G$ be a connected semi-simple (L)-group, and $C$ the maximal compact connected invariant subgroup of $G$. Then there exists a closed connected invariant Lie subgroup $p^{\circ} L$ of $G$ so that $G=C L$, and $[C, L]=\{e\}$, and that the intersection of $C$ and $L$ is a finite group.

(2) Any factor group of $G$ is semi-simple.

Using the latter part of Theorem 1, we can easily prove the following corollary:

COROLlary 1. Let $G$ be a connected ( $L$ )-group and $R$ the radical of $G$. If $N$ is a closed invariant subgroup of $G$ such that $R N$ is closed, then $R N / N$ is the radical of $G / N$.

Corollary 2. Let $G$ be a connected group and $S$ a closed connected invariant subgroup. We denote by $S^{\perp}$ the centralizer ${ }^{10}$ of $S$. If $S$ is a semi-simple $(L)$-group, then we have $G=S S^{\perp}$.

Proof. Corollary 2 has been proved in [K.I.] for any compact invariant subgroup $S$. It is also clear for a connected semi-simple Lie group $S$. Now it is not hard to establish the corollary from these special cases. Q.E.D.

2. Proof of Theorem 2. In this section we shall give a proof of the following theorem.

TheOREM 2. Let $G$ be an (l)-group and $K$ a compact invariant subgroup. Then the factor group $G / K$ is also an (l)-group.

${ }^{8}$ Cf. H. Freudenthal, Topologische Gruppen mit genilgend vielen fastperiodischen Funktionen, Ann. of Math. vol. 37 (1936).

- By a (closed) Lie subgroup we mean a subgroup which is a Lie group.

${ }^{10}$ Namely, the (invariant) subgroup composed of all elements which commute with every element of $S$. 
In order to prove Theorem 2, we first recollect and modify some results stated in [M.G.].

Let $G$ be an f.r. group, and $N$ a maximal closed simply connected solvable invariant subgroup of $G$. Then there exists a closed subgroup $T$, whose radical is compact, such that

$$
G=T N, \quad T \cap N=\{e\} .
$$

Conversely a connected Lie group is f.r. if it admits such a decomposition. ${ }^{11}$

Now the radical of an f.r. group is compact if and only if it is completely reducible, ${ }^{12}$ where the complete reducibility of an (l)group is defined as follows:

Definition 3. An (b)-group $G$ is called completely reducible if every representation of $G$ is completely reducible.

Let $T$ be a completely reducible f.r. group and $A$ the radical of $T$. Then $A$ is compact and there exists in $T$ a closed connected semisimple subgroup $S$ such that $T=S A$. It is clear that $[S, A]=\{e\}$ and the intersection of $S$ and $A$ is a finite group. Now any factor group of a semi-simple f.r. group is also f.r., ${ }^{18}$ and a connected Lie group is f.r. if its radical and a maximal semi-simple subgroup ${ }^{14}$ are f.r. ${ }^{15}$ These obviously imply that a factor group of a completely reducible f.r. group is also a completely reducible f.r. group.

Next, it is to be remarked that a simply connected solvable Lie group is homeomorphic with the Euclidean space, and that a locally compact connected solvable group is a simply connected Lie group if and only if it contains no compact subgroup other than the identity group. ${ }^{16}$

Now the following lemma is a special case of Theorem 2.

LEMMA 3. Let $G$ be an f.r. group and $K$ a compact invariant subgroup. Then the factor group $G / K$ is also f.r.

Proof. Let $G=T N$ be a decomposition as above, and let $M$ be a maximal compact subgroup ${ }^{17}$ of $T$. Since $N$ is homeomorphic with

11 [M.G.II, Corollary 2 to Theorem 7].

12 [M.G.II, Lemma 14].

13 [M.G.II, Lemma 9].

${ }^{14}$ By a maximal semi-simple subgroup of a Lie group we mean a connected group generated by a closed maximal semi-simple local subgroup.

15 [M.G.II, Theorem 7]. A. Malcev, On linear Lie groups, C.R. (Doklady) Acad. Sci. URSS. (1943).

${ }^{16}$ See [K.I.].

${ }^{17}$ See [K.I.]. 
the Euclidean space, $M$ is maximal compact also in $G$. Hence $K$ is contained in $M$, and thus we have $K \subset T$. Now since $T$ is completely reducible, the factor group $T / K$ is a completely reducible f.r. group. It is now clear that $G / K$ is f.r., because

$$
G / K=T / K \cdot(N K) / K
$$

is also a decomposition as above. Q.E.D.

Proof of Theorem 2. Let $g$ be an element of $G$ which is not contained in $K$. For any element $x$ of $K$ we can readily select a representation $f_{x}$ of $G$ and a neighborhood $U_{x}$ of $x$ such that $f_{x}\left(U_{x} g\right)$ does not contain the unit matrix. Now there exists a finite system $\left\{x_{1}, \cdots, x_{n}\right\}$ of elements of $K$ such that the system $\left\{U_{x_{1}}, \cdots, U_{x_{n}}\right\}$ of neighborhoods covers $K: \cup U_{x_{i}} \supset K$.

Now let $f$ be the so-called sum representation of $f_{x_{1}}, \cdots, f_{x_{n}}$, and let $N_{1}, N_{2}, \cdots, N_{n}, N$ be the kernel of $f_{x_{1}}, f_{x_{2}}, \cdots, f_{x_{n}}, f$. From the relations $U_{x_{i}} g \cap N_{i}=0(i=1,2, \cdots, n)$, and $\cap N_{i}=N$, we have $U_{x_{i}} g \cap N=0$, where 0 denotes the empty set. Accordingly we obtain $K g \cap N=0$, whence

$$
K g \cap K N=0 .
$$

Now since $G / N$ is f.r. and $K N / N$ is a compact invariant subgroup of $G / N, G / K N$ is also f.r. by Lemma 3. Now the relation $K g \cap K N=0$ implies that $\mathrm{Kg} / \mathrm{K}$ is not contained in the kernel of the homomorphism $G / K \sim G / K N: G / K / K N / K \cong G / K N$, and this completes our proof.

CoRollary. Let $G$ be an (l)-group, and $U$ a neighborhood of the identity in $G$. Then there exists a compact invariant subgroup $K$, which is contained in $U$, such that the factor group $G / K$ is f.r.

Proof. Since $G$ is a connected $(L)$-group, there exists a compact invariant subgroup $K$ such that $G / K$ is a Lie group and $K \subset U$, in virtue of [K.I.]. Thus the corollary is an immediate consequence of Theorem 2. Q.E.D.

3. Completely reducible (l)-groups. In [M.G. II ${ }^{18}$ we have determined the class of all semi-simple f.r. groups. Here we consider semi-simple (l)-groups.

Theorem 3. (1) Let $G$ be a semi-simple (L)-group, and let

$$
G=C L
$$

${ }^{18}$ Lemma 9, Theorem 3, and Theorem 4. 
be the decomposition of $G$ as in Theorem 1 . Then $G$ is an (l)-group if and only if $L$ is f.r.

(2) The center of a semi-simple (l)-group is compact.

(3) Any factor group of a semi-simple (l)-group is also an (l)-group.

Proof. Assume that $L$ be f.r. Then the direct product $C \times L$ is clearly an $(l)$-group. Now we can readily find a finite central invariant subgroup $D$ of $C \times L$ such that $C \times L / D \cong G$. Hence $G$ is an (l)group by Theorem 2 . The converse is trivial.

Next, we know that the center of a semi-simple f.r. group is finite. ${ }^{19}$ From this the second assertion follows immediately.

Further, let $G$ be a semi-simple ( $l$ )-group and $N$ a closed invariant subgroup of $G$. We denote by $N^{0}$ the connected component of $N$ containing the identity. $N^{0}$ is clearly a semi-simple (l)-group. Let $C^{0}$ be the maximal connected compact invariant subgroup of $N^{0}$ and let

$$
N^{0}=C^{0} L^{0}
$$

be the decomposition of $N^{0}$ as in Theorem 1. $C^{0}$ is obviously an invariant subgroup of $G$, and the compactness of $C^{0}$ implies that $G / C^{0}$ is an (l)-group by Theorem 2. Let now $G / C^{0}=C^{*} L^{*}$ be the decomposition of $G / C^{0}$ as in Theorem 1 . Then the invariant subgroup $N^{0} / C^{0}$ is clearly contained in $L^{*}$. The fact that a factor group of a semisimple f.r. group is f.r. readily implies that $G / N^{0}$ is also an (l)-group. Hence the center of $G / N^{0}$ is compact by our above assertion. This shows the compactness of $N / N^{0}$, and we obtain the last assertion, using Theorem 2 again. Q.E.D.

It is well known that a semi-simple f.r. group is completely reducible, and so is a semi-simple ( $l$-group. Now, we study general completely reducible (l)-groups. A criterion for the complete reducibility is furnished by the following lemma.

Lemma 4. An (l)-group is completely reducible if and only if its radical is compact.

Proof. Let $G$ be a completely reducible (l)-group. According to the corollary of Theorem 2, there exists a compact invariant subgroup $K$ such that $G / K$ is f.r. Denote by $R$ the radical of $G$. Then $R K / K$ is the radical of $G / K$, in virtue of Corollary 1 to Theorem 1 . Now since $G / K$ is obviously completely reducible, its radical $R K / K$ is compact. Hence $R$ must be compact.

Conversely, let $G$ be an (l)-group with the compact radical $R$, and let $N$ be an arbitrary closed invariant subgroup of $G$ such that the

10 [M.G.I, Lemma 5]. 
factor group $G / N$ is f.r. It is sufficient to show that $G / N$ is completely reducible, or what is the same, that the radical of $G / N$ is compact. Now the compactness of $R$ implies that $R N / N$ is the compact radical, and this completes our proof.

Let $G$ be a topological group. We denote by $d(G)$ the commutator group of $G$, that is, $[G, G]$, and by $D(G)$ the closure of $d(G)$. Following Iwasawa we call $D(G)$ the topological commutator group of $G$. The topological commutator group of a connected group is obviously connected.

Next, let $G$ be a completely reducible ( $l$ )-group and $R$ the radical of $G$. Since $R$ is compact, $D(G) R / D(G)$ is the radical of the commutative $G / D(G)$, Hence $D(G) R$ coincides with $G: G=D(G) R$. Now let $K$ be a compact invariant subgroup of $G$. Since it is clear that $\overline{d(G) K}$ $=D(G) K$, we have

$$
D(G) K / K=D(G / K) .
$$

Let $Z$ be the intersection of $D(G)$ and $R$. In any representation of $G$ with a compact kernel, $Z$ goes over into the intersection of the commutator group and the radical, which is obviously a finite group. Hence $Z$ must be 0 -dimensional. That $D(G)$ is semi-simple follows immediately.

Now it is easy to prove the following theorem.

TheOREM 4. (1) Let $G$ be a completely reducible (l)-group, and $C$ the maximal connected compact invariant subgroup. $G$ contains a closed connected semi-simple f.r. invariant subgroup $L$ such that $G=C L$, $[C, L]=\{e\}$, and the intersection of $C$ and $L$ is a finite group. And conversely a topological group which admits such a decomposition is a completely reducible (l)-group.

(2) Any solvable invariant subgroup of a completely reducible (l)group is contained in the center, which is compact.

(3) Any factor group of a completely reducible (l)-group is also a completely reducible (l)-group.

REMARK. It is well known that a locally compact connected group is maximally almost periodic if and only if it is decomposable into a direct product of a compact group and a vector group. ${ }^{20}$ On the other hand, it is clear that a vector group is not completely reducible. Hence, or from Theorem 4, we get the following proposition, by which the position of compact groups in the theory of topological groups is clarified:

${ }^{20} \mathrm{H}$. Freudenthal, loc. cit. 
A locally compact connected group is a completely reducible, maximally almost periodic group if and only if it is compact.

\section{Fundamental theorems.}

THEOREM 5. Let $G$ be a locally compact connected group. If $G$ is an (l)-group, then there exists a closed completely reducible (l)-group $T$ in $G$ and a closed simply connected solvable Lie invariant subgroup $N$ such that

$$
G=T N, \quad T \cap N=\{e\}
$$

and conversely.

Proof. Let $G$ be an ( $l$ )-group. In virtue of the corollary to Theorem 2 , we can select a sequence $\left\{K_{\alpha}\right\}$ of compact invariant subgroups of $G$ such that $G_{\alpha}=G / K_{\alpha}$ is f.r. and the intersection of all $K_{\alpha}$ 's coincides with the identity group. Now, because of the compactness of $K_{\alpha}$, there corresponds $D\left(G_{\alpha}\right)$ to $D(G)$ in the homomorphism $G \sim G_{\alpha}$.

We denote by $M$ the radical of $D(G)$. Since $K_{\alpha}$ is compact, $M K_{\alpha} / K_{\alpha}$ is the radical of $D\left(G_{\alpha}\right)$. On the other hand we know that the radical of the commutator group of an f.r. group is simply connected.21 Hence $M$ cannot contain a compact subgroup other than the identity group. Therefore $M$ is a simply connected Lie group.

Now, since $D(G)$ is an (l)-group by itself, $D(G)$ contains a compact invariant subgroup $K$ such that the factor group $D(G)^{*}=D(G) / K$ is f.r. Now since $M \cap K=\{e\}$, and $M K$ is closed, $M^{*}=M K / K$, which is isomorphic with $M$, is the radical of $D(G)^{*}$. Let $S^{*}$ be a maximal semi-simple subgroup of $D(G)^{*}$. The center of $S^{*}$ is finite and $S^{*}$ is closed. Hence the intersection of $S^{*}$ and $M^{*}$ is the identity group. Thus we obtain the decomposition

$$
D(G)^{*}=S^{*} M^{*}, \quad S^{*} \cap M^{*}=\left\{e^{*}\right\},
$$

where $e^{*}$ denotes the identity of $D(G)^{*}$.

Now let $S$ be the complete inverse image of $S^{*}$ in the homomorphism $D(G) \sim D(G)^{*}$. Then $S$ is obviously semi-simple and we get

$$
D(G)=S M \text {. }
$$

Since any solvable invariant subgroup of $S$ is easily seen to be compact, we have

$$
S \cap M=\{e\} .
$$

${ }^{21}$ See [M.G.I, Lemma 7]. 
Next, since $G / D(G)$ is connected and commutative, using the structure theorem of Pontrjagin, we can assure the existence of a closed invariant subgroup $H$ containing $D(G)$ such that $G / H$ is compact and $H / D(G)$ is a vector group.

We denote by $N$ the radical of $H$. Now we select a compact invariant subgroup $K_{1}$ of $H$ so that $H / K_{1}$ is f.r. $K_{1}$ is obviously contained in $D(G)$. Hence we can consider $K_{1}$ as $K$ mentioned above and retain the above notations. Let $N^{*}$ be the image of $N$ in the homomorphism $H \sim H^{*}=H / K . N^{*}$ is clearly the radical of $H^{*}$. The fact that $H^{*} / D(G)^{*}$ is commutative immediately implies that any maximal semi-simple subgroup of $H^{*}$ is contained in $D(G)^{*}$. Hence $S^{*}$, as a maximal semi-simple subgroup of $D(G)^{*}$, is also a maximal semi-simple subgroup in $H^{*}$. Thus we have $H^{*}=S^{*} N^{*}$. Hence we get that

$$
H=S N .
$$

Now $M^{*}$ is obviously locally coincident with $D(G)^{*} \cap N^{*}$. On the other hand from the relation

$$
H^{*} / D(G)^{*} \cong N^{*} / D(G)^{*} \cap N^{*},
$$

$N^{*} / D(G)^{*} \cap N$ is a vector group. This is impossible unless $D(G)^{*} \cap N^{*}$ is connected. Hence we have $D(G)^{*} \cap N^{*}=M^{*}$. Thus the simple connectedness of $M^{*}$ and that of $N^{*} / M^{*}$ imply that of $N^{*}$.

Next we shall prove that $N$ is a simply connected Lie group isomorphic with $N^{*}$. First, since the center of $S$ is compact, $S \cap N$ must be a compact 0 -dimensional group. Hence $K \cap N$ is also compact 0 -dimensional. Now suppose that $K \cap N \neq\{e\}$. Then there exists a proper open (closed) subgroup $Q$ of $K \cap N$. Then by the relation $N / K \cap N \cong N^{*}, N / Q$ is a proper covering group of $N^{*}$. This is clearly impossible, because $N^{*}$ is simply connected. Hence $K \cap N=\{e\}$. Thus our assertion holds immediately, and moreover we get

$$
S \cap N=\{e\} .
$$

Next we put $G / N=G^{\prime}, H / N=H^{\prime}$. Then $G^{\prime}$ is an (L)-group, and $H^{\prime}$ is a closed connected semi-simple invariant subgroup of $G^{\prime}$ because $H^{\prime} \cong S$. Hence by Corollary 2 to Theorem 1 there exists a closed invariant subgroup $A_{1}^{\prime}$ of $G^{\prime}$ so that $G^{\prime}=H^{\prime} A_{1}^{\prime}$ and $\left[H^{\prime}, A_{1}^{\prime}\right]$ $=\left\{e^{\prime}\right\}$, where $e^{\prime}$ is the identity of $G^{\prime}$. The center of $H^{\prime}$ coincides with $H^{\prime} \cap A_{1}^{\prime}$, and is compact because $H^{\prime}$ is a semi-simple (l)-group. On the other hand, the relation $G / H \cong G^{\prime} / H^{\prime} \cong A_{1}^{\prime} / H^{\prime} \cap A_{1}^{\prime}$ implies that $A_{1}^{\prime} / H^{\prime} \cap A_{1}^{\prime}$ is compact. Hence $A_{1}^{\prime}$ is a compact group. Let now $A^{\prime}$ be the connected component of $A_{1}^{\prime}$ containing $e^{\prime}$. Then Lemma 1 
implies that $G^{\prime}=H^{\prime} A^{\prime}$.

Next we denote by $R$ the complete inverse image of $A^{\prime}$ in the homomorphism $G \sim G^{\prime} . R$ is clearly the radical of $G$, and we get

$$
G=H R=S R \text {. }
$$

Let now $G \sim(G)$ be a homomorphism of $G$ onto an f.r. group $(G)$ such that the kernel is compact, and let $(R),(N)$ be the image of $R, N$ respectively in the homomorphism. It is clear that $(R)$ is the radical of $(G)$ and $(N)$ a simply connected Lie group such that $(R) /(N)$ is compact. Hence $(N)$ is a maximal closed simply connected solvable invariant subgroup of $(G)$. Now since $(G)$ is f.r., $(G)$ contains a closed completely reducible f.r. group $(T)$ such that

$$
(G)=(T)(N), \quad(T) \cap(N)=\{(e)\} .
$$

Let $T$ be the complete inverse image of $(T)$ in $G$. The fact that $N$ contains no compact subgroup other than $\{e\}$ readily implies the following relations

$$
G=T N, \quad T \cap N=\{e\} .
$$

Thus we obtain in particular the connectedness of $T$. Now it is easy to prove that the radical of $T$ is compact. Hence $T$ is completely reducible by Lemma 4 . Thus our first assertion is proved.

Conversely, let $G$ be a locally compact connected group which admits a decomposition $G=T N, T \cap N=\{e\}$, as above. Then, since $G / N \cong T$ and $T$ is an (l)-group, there exists a system $\left\{N_{\alpha}\right\}$ of closed invariant subgroups containing $N$ such that $\cap N_{\alpha}=N$ and $G / N_{\alpha}$ 's are f.r.

Now let us denote by $T_{1}$ the set of all elements of $T$ which are commutative with every element of $N$. Then $T_{1}$ is clearly a closed invariant subgroup of $G$ such that $T / T_{1}$ is f.r. Now in virtue of Theorem $4, T / T_{1}$ is completely reducible. Hence the relations

$$
\begin{gathered}
G / T_{1}=T / T_{1} \cdot N T_{1} / T_{1}, \quad T / T_{1} \cap N T_{1} / T_{1}=T_{1} / T_{1}, \\
N T_{1} / T_{1} \cong N,
\end{gathered}
$$

imply that $G / T_{1}$ is f.r. Thus because of the relation $\cap N_{\alpha} \cap T_{1}=\{e\}$ we get the result. Q.E.D.

Corollary. Let $G$ be an (l)-group and $K$ a maximal compact subgroup of $G$. Then there exists a closed connected simply connected solvable Lie subgroup $H$ of $G$ such that

$$
G=H K=K H, \quad K \cap H=\{e\} .
$$


Proof. In virtue of Theorem 5 , it is clearly sufficient to consider the case when $G$ is completely reducible. On the other hand, the corollary holds for f.r. groups by [M.G.II]. ${ }^{22}$ Thus by Theorem 4 we can readily complete our proof. Q.E.D.

Theorem 6. Let $G$ be a locally compact connected group and $R$ the radical of $G$. If $R$ is an (l)-group and $G$ contains a closed semi-simple (l)-group $S$ such that

$$
G=S R,
$$

then $G$ is an (l)-group, and conversely.

PRoof. In the first place we suppose that $R$ is an (l)-group and $S$ is a closed semi-simple (l)-group in $G$ such that $G=S R$. We can prove that $G$ is an $(L)$-group because $G / R$ is obviously an $(L)$-group. ${ }^{23}$ Let $K$ be a compact invariant subgroup of $G$ such that $G / K$ is a Lie group. Then $S K / K$ is clearly a maximal semi-simple subgroup, and $R K / K$ the radical, of $G / K$. Since they are f.r. by Theorem $2, G / K$ itself is also f.r. in virtue of [M.G.II]. This means that $G$ is an (l)-group.

Conversely, let $G$ be an (l)-group and $G=T N$ a decomposition as in Theorem 5. Let $D(T)$ be the topological commutator group of $T$, and $A$ the radical of $T$. Then $D(T)$ is semi-simple and we get $T$ $=D(T) A$ by Theorem 4 . Now it is clear that $A N$ coincides with the radical $R$. Hence we have $G=D(T) R$, and this completes our proof.

5. Solvable (l)-groups. Let $G$ be a locally compact connected solvable group. Then $G$ is an $(L)$-group in virtue of [K.I.]. Hence there exists a compact invariant subgroup $K$ such that $G^{*}=G / K$ is a Lie group. Let now $D(G)$ be the topological commutator group of $G$. Then because of the compactness of $K$ we have

$$
D\left(G^{*}\right)=D(G)^{*}(=D(G) K / K) .
$$

Now it is well known that $D(G)^{*}$ is nilpotent. On the other hand $K$ is a central invariant subgroup by [K.I.]. Hence $D(G) K$ is nilpotent, and so is $D(G)$. Thus the topological commutator group of a locally compact connected solvable group is nilpotent. ${ }^{24}$

22 [M.G.II, Corollary 1 to Theorem 7].

${ }^{23}$ Let $G$ be a locally compact connected group, and $N$ a closed connected invariant subgroup of $G$. Suppose $N$ and $G / N$ are both (L)-groups. Then $G$ is an ( $L$ )-group by itself. See [K.I.].

${ }^{24}$ We can prove moreover the following theorem: The radical of the topological commutator group of a connected $(L)$-group is nilpotent. This is an extension of a theorem of E. Cartan. Cf. E. Cartan, Thèse, Paris, 1894. 
Now we shall prove the following lemma.

LEMMA 5. Let $G$ be a connected group and $N$ a closed connected invariant subgroup. Let $K$ be a compact subgroup of $N$. If $N$ is locally compact and nilpotent, then $K$ is central in $G$.

Proof. Let $K_{1}$ be the maximal compact invariant subgroup of $N$. Then $K_{1}$ is commutative and $N / K_{1}$ is a Lie group. On the other hand, we know that any compact subgroup of a connected nilpotent Lie group is a central invariant subgroup. ${ }^{25}$ Hence we can easily prove that $K$ is contained in $K_{1}$.

Now it is clear that $K_{1}$ is an invariant subgroup of $G$. Then because of the connectedness of $G, K_{1}$ must be central. ${ }^{26}$ Hence $K_{1}$ is a central invariant subgroup of $G$. Q.E.D.

It is now easy to establish the following theorem.

THEOREM 7. Let $G$ be a locally compact connected solvable group and $A$ a maximal compact subgroup of $G$. The following conditions are all necessary and sufficient for $G$ to be an (l)-group. (1) $G=A N, A \cap N$ $=\{e\}$, where $N$ is a closed simply connected Lie invariant subgroup. (2) $D(G)$ is a simply connected Lie group. (3) The center of $D(G)$ is a connected simply connected Lie group. (4) $A \cap D(G)=\{e\}$.

From Lemma 5 and Theorem 7 we have in particular the following corollary.

CoRollary. A locally compact connected nilpotent group is an (l)group if and only if it is a direct product of a compact group and a connected simply connected Lie group.

NAGOYA UNIVERSITY

25 [M.G.II, Lemma 13].

${ }^{26}$ See [K.I.]. 